Pacific Journal of Mathematic 


\section{THE DUNFORD-PETTIS PROPERTY FOR CERTAIN UNIFORM ALGEBRAS}

\section{F. Delbaen}

A Banach space $B$ has the Dunford-Pettis property if $x_{n}^{*}\left(x_{n}\right) \rightarrow 0$ whenever $x_{n} \rightarrow 0$ weakly and the sequence $x_{n}^{*}$ tends to zero weakly in $B^{*}$ (i.e. $\sigma\left(B^{*}, B^{* *}\right)$ ). Suppose now that $A$ is a uniform algebra on a compact space $X$. If $\phi$ is a nonzero multiplicative linear functional on $A$ then $M_{\phi}$ is the set of positive representing measures of $\phi$. If $A$ is such that a singular measure which is orthogonal to $A$ must necessarily be zero and if all $M_{\phi}$ are weakly compact sets then the algebra $A$ as well as its dual have the Dunford-Pettis property.

The idea of the proof is that $A^{*}$ the dual of $A$ can be decomposed into components for which the results of Chaumat [1] and Cnop-Delbaen [2] can be applied. The fact that an $l_{1}$ sum of DunfordPettis spaces is also a Dunford-Pettis space then gives the result. In paragraph two some conditions ensuring the weak compactness of $M_{\dot{\phi}}$ are given. These conditions are related to those used in the definition of core and enveloping measures (see [6]).

1. Notation and preliminaries. $X$ will be a compact space, $A \subset \mathscr{C}(X)$ a closed subalgebra of the space of continuous complexvalued functions on $X$. The algebra $A$ is supposed to contain the constants and to separate the points of $X$. The spectrum $M_{A}$ is the set of all nonzero multiplicative linear functionals on $A$. If $\phi \in M_{A}$ then $M_{\phi}$ is the set of all positive measures on $X$ representing $\phi$, i.e.

$$
M_{\phi}=\left\{\mu \in M(X) \mid \mu \geqq 0 \text { and } \forall f \in A \text { we have } \phi(f)=\int f d \mu\right\} .
$$

As well known $M_{\phi}$ is a convex set, compact for the topology $\sigma(M(X), \mathscr{C}(X))$. We say that two multiplicative linear forms $\phi$ and $\psi$ belong to the same Gleason part if $\|\phi-\psi\|<2$ in $A^{*}$, the dual of $A$. It is well known that being in the same Gleason part is an equivalence relation and hence $M_{A}=\mathrm{U}_{x \in \Pi} \pi$ where $\Pi$ is the set of all Gleason equivalence classes. For more details and any unexplained notion on uniform algebras we refer to [6].

If $E$ is a Banach space then $E$ has the Dunford-Pettis property if $e_{n}^{*}\left(e_{n}\right) \rightarrow 0$ whenever $e_{n} \rightarrow 0$ weakly and $e_{n}^{*} \rightarrow 0$ weakly (i.e. $\left.\sigma\left(E^{*}, E^{* *}\right)\right)$.

For more details and properties of such spaces see Grothendieck 
[4] or [5], where it is also proved that $L^{1}$ spaces and $\mathscr{C}(X)$ spaces have the Dunford-Pettis property.

2. Weak compactness of $M_{\phi}$. We investigate under what conditions $M_{\phi}$ is weakly compact. First we remark that if $\psi$ and $\phi$ are in the same Gleason part then there is an affine isomorphism linking $M_{\phi}$ and $M_{\psi}$, see [6, p. 143]. It follows that $M_{\phi}$ is weakly compact (i.e. $\sigma\left(M(X), M(X)^{*}\right)$ ) if and only if $M_{\psi}$ is weakly compact. Moreover if $m_{\phi}$ is dominant in $M_{\dot{\phi}}$ and $m_{\psi}$ is dominant in $M_{\psi}$ then $m_{\phi}$ is absolutely continuous with respect to $m_{\psi}$. (The existence of a dominant measure in $M_{\phi}$ is given by [3, p. 307].)

LEMma. If $\phi$ is an element of $M_{A}$ then following are equivalent 1. $M_{\dot{\phi}}$ is weakly compact.

2. If $u_{n}$ is a sequence of continuous functions on $X$ such that $1 \geqq u_{n} \geqq 0$ and $u_{n} \rightarrow 0$ pointwise then there is a subsequence $n_{k}$ and functions $v_{k} \in A$ such that Re $v_{k} \geqq u_{n_{k}}$ and $\phi\left(v_{k}\right) \rightarrow 0$.

3. If $u_{n}$ is a sequence of continuous functions on $X$ such that $1 \geqq u_{n} \geqq 0$ and $u_{n} \rightarrow 0$ pointwise then there is a subsequence $n_{k}$ and functions $g_{k} \in A$ such that $\left|g_{k}\right| \leqq e^{-u_{n_{k}}}$ and $\phi\left(g_{k}\right) \rightarrow 1$.

Proof. (1) $\Rightarrow$ (2) If $M_{\phi}$ is weakly compact and $u_{n}$ is a sequence as in (2) then $\sup _{\mu \in M_{\phi}} \int u_{n} d \mu \rightarrow 0$ (see [4]). Hence if $\varepsilon_{n}$ is a sequence of strictly positive numbers tending to zero then $\exists v_{n} \in A$ such that $\operatorname{Re} v_{n} \geqq u_{n}$ and $\phi\left(v_{n}\right) \leqq \sup _{\mu \in M_{\phi}} \int u_{n} d \mu+\varepsilon_{n} \quad$ (see [ [ $\begin{array}{lll}6 & \text { p. 82]). Clearly }\end{array}$ $\phi\left(v_{n}\right) \rightarrow 0$.

(2) $\Rightarrow$ (3) Write $g_{k}=e^{-v_{k}}$ and observe that $\left|g_{k}\right|=e^{-\operatorname{Re} v_{k}} \leqq e^{-u_{n_{k}}}$ and $\phi\left(g_{k}\right)=e^{-\phi\left(v_{k}\right)} \rightarrow 1$.

(3) $\Rightarrow$ (1) If $M_{\dot{\phi}}$ is not weakly compact then following [4] there is a sequence of functions $u_{n} \in \mathscr{C}(X)$ and a sequence of measures $\mu_{n} \in M_{\phi}$ as well as $\varepsilon>0$ such that

(i) $0 \leqq u_{n} \leqq 1$ and $u_{n} \rightarrow 0$ pointwise

(ii) $\int u_{n} d \mu_{n}>\varepsilon$.

Let now $g_{k}$ be as in (3) then

$$
\left|\phi\left(g_{k}\right)\right| \leqq \int\left|g_{k}\right| d \mu_{n_{k}} \leqq \int e^{-u_{n_{k}}} d \mu_{n_{k}} \leqq 1-\frac{e-1}{e} \int u_{n_{k}} d \mu_{n_{k}} \leqq 1-\frac{e-1}{e} \varepsilon
$$

and this contradicts $\phi\left(g_{k}\right) \rightarrow 1$.

REMARK. The conditions (2) and (3) are of course related to the conditions of being enveloped and being a core measure. The dif- 
ference is that the sequence $u_{n}$ is supposed to be uniformly bounded.

Corollary. If A satisfies one of the following conditions then for all $\phi \in M_{A}, M_{\phi}$ is weakly compact.

(1) If $1 \geqq u_{n} \geqq 0 ; u_{n} \in \mathscr{C}(X)$ and $u_{n} \rightarrow 0$ pointwise then there $i$ s a subsequence $n_{k}$ and $v_{k} \in A$ such that $v_{k}$ are uniformly bounded, $\operatorname{Re} v_{k} \geqq u_{n_{k}}$ and $v_{k} \rightarrow 0$ on $X$.

(2) If $1 \geqq u_{n} \geqq 0 ; u_{n} \in \mathscr{C}(X)$ and $u_{n} \rightarrow 0$ pointwise then there is a subsequence $n_{k}$ and $g_{k} \in A$ such that $\left|g_{k}\right| \leqq e^{-u_{n_{k}}}$ and $g_{k} \rightarrow 1$ on $X$.

3. The D.P. property for some uniform algebras. In the following theorem we say that a measure $\nu$ is singular to $A$ if for all $\phi$ and all $\mu \in M_{\phi}$, the measure $\nu$ is singular with respect to $\mu$.

THEOREM. A has the Dunford-Pettis property if

(1) for all $\phi \in M_{A}$, the set $M_{\phi}$ is weakly compact,

(2) if $\lambda$ is orthogonal to $A$ and $\lambda$ is singular to $A$ then $\lambda=0$.

Proof. Of course we only have to prove that $A^{*}$ has the D.P. property, since it follows from the definition that a Banach space is a Dunford-Pettis space as soon as its dual is a Dunford-Pettis space. We first prove the following lemma.

Lemma. If $\left(E_{\beta}\right)_{\beta \in B}$ is a family of Banach spaces all having the D.P. property and if

$$
\left(\sum_{\beta} \oplus E_{\beta}\right)_{l_{1}}=E=\left\{e=\left(e_{\beta}\right)_{\beta \in B} \mid e_{\beta} \in E_{\beta} ; \sum_{\beta}\left\|e_{\beta}\right\|=\|e\|<\infty\right\}
$$

then $E$ has the D.P. property.

Proof. $\forall \beta$ let $P_{\beta}: E \rightarrow E_{\beta}$ be the canonical projection.

Let $e_{n} \in E$ such that $e_{n} \rightarrow 0$ weakly and $\left\|e_{n}\right\| \leqq 1 ; e_{n}^{*} \in E^{*}$ such that $e_{n}^{*} \rightarrow 0$ weakly and $\left\|e_{n}^{*}\right\| \leqq 1 ; P_{\beta} e_{n}=e_{n, \beta} ; P_{\beta}^{*} e_{n}^{*}=e_{n, \beta}^{*} ; t_{n, \beta}=$ $e_{n, \beta}^{*}\left(e_{n, \beta}\right)$.

Only a denumerable part of the numbers $t_{n, \beta}$ can be different from zero so we can take $B=N$. We first prove that the sum $e_{n}^{*}\left(e_{n}\right)=\sum_{\beta} t_{n, \beta}$ converges uniformly in $n$, i.e.

(*) for all $\varepsilon>0$ there is $N$ such that $\forall n$ we have $\sum_{\beta>N}\left|t_{n, \beta}\right|<\varepsilon$. If this is not the case then we start a well-known procedure. Let $\varepsilon>0$ be such that $(*)$ does not hold for this $\varepsilon$, take $\delta_{n}>0$ such that $\sum_{n=1}^{\infty} \delta_{n} \leqq \varepsilon / 4$. Let $n_{1}=1, N_{0}=0, N_{1}$ such that $\sum_{\beta>N_{1}}\left\|e_{n_{1}, \beta}\right\| \leqq \delta_{1}$.

Since $e_{n, 1}, \cdots, e_{n, N_{1}} \rightarrow 0$ weakly we can find $\bar{n}_{2}$ such that for all $n \geqq \bar{n}_{2} \geqq n_{1}$ we have $\sum_{\beta=1}\left|e_{n, j}^{*}\left(e_{n_{1}, j}\right)\right| \leqq \delta_{2}$. Let now $n_{2} \geqq \bar{n}_{2}$ be such 
that $\sum_{\beta>N_{1}}\left|t_{n_{2}, \beta}\right|>\varepsilon$ and $N_{2}>N_{1}$ such that $\sum_{\beta>N_{2}}\left\|e_{n_{2}, \beta}\right\| \leqq \delta_{2}$. Continuing this procedure we find two strictly increasing sequences $\left(n_{k}, N_{k}\right)$ such that

(1) $\sum_{\beta>N_{k}}\left\|e_{n_{k}, \beta}\right\| \leqq \delta_{k}$

(2) $\forall n \geqq n_{k}$ the $\operatorname{sum} \sum_{\beta=1}^{N_{k-1}}\left|e_{n, j}^{*}\left(e_{n_{k-1}, \beta}\right)\right| \leqq \delta_{k}$

(3) $\sum_{\beta>N_{k-1}}\left|t_{n_{k}, \beta}\right|>\varepsilon$.

Let now

$$
e^{*}=\left(\gamma_{1} e_{1,1}^{*} ; \cdots: \gamma_{N_{1}} e_{1, N_{1}}^{*} ; \gamma_{N_{1}+1} e_{n_{2}, N_{1}+1}^{*} ; \cdots ; \gamma_{N_{2}} e_{N_{2}}^{*} ; \gamma_{N_{2}+1} e_{n_{3}, N_{2}+1}^{*} ; \cdots\right)
$$

where $\gamma_{\beta}$ is such that if $N_{k-1}+1 \leqq \beta \leqq N_{k}$ then $\gamma_{\beta} e_{n_{k}, \beta}^{*}\left(e_{n_{k}, \beta}\right)=\left|t_{n_{k}, \beta}\right|$. Clearly $e^{*} \in E^{*}$ and $\left\|e^{*}\right\| \leqq 1$. For all $k \geqq 2$

$$
\begin{aligned}
e^{*}\left(e_{n_{k}}\right)= & \sum_{j=1}^{k-1} \sum_{\beta=N_{j-1}+1}^{N_{j}} \gamma_{\beta} e_{n_{j}, \beta}^{*}\left(e_{n_{j}, \beta}\right) \\
& +\sum_{\beta=N_{k-1}+1}^{N_{k}}\left|t_{n_{k}, \beta}\right| \\
& +\sum_{\beta>N_{k}} \gamma_{\beta} e_{\beta}^{*}\left(e_{n_{k}, \beta}\right) .
\end{aligned}
$$

So

$$
\begin{aligned}
\left|e^{*}\left(e_{n_{k}}\right)\right| & \geqq-\sum_{j=1}^{k-1} \delta_{j}+\sum_{\beta=N_{k-1}+1}^{N_{k}}\left|t_{n_{k}, \beta}\right|-\delta_{k} \\
& \geqq-\sum_{j=1}^{k} \delta_{j}+\sum_{\beta>N_{k-1}}\left|t_{n_{k}, \beta}\right|-2 \delta_{k} \\
& \geqq \varepsilon-2_{j=1}^{\infty} \sum \delta_{j} \geqq \varepsilon / 2 .
\end{aligned}
$$

But this contradicts $e_{n_{k}} \rightarrow 0$ weakly. This proves that $(*)$ is verified and hence $\lim _{n \rightarrow \infty} \sum_{\beta} t_{n, \beta}=\sum_{\beta} \lim t_{n, \beta}=0$, since each of the $E_{\beta}$ has the D.P. property.

REMARK. If $E_{n}=l_{2}^{n}$ (i.e. the $n$-dimensional Hilbert space) then $E=\left(\Sigma \oplus E_{n}\right)_{l_{1}}$ has the D.P. property but $E^{*}$ has not, because as easily seen, the space $E^{*}$ has a complemented subspace isometric to $l_{2}$, this contradicts D.P. (see [4]).

Proof of the theorem. For each $\pi \in \Pi$ we select $\phi_{\pi} \in \pi$ and $m_{\pi} \in M_{\phi}$ dominant. By [6 p. 144] all $m_{\pi}$ are mutually singular. Select now probability measures $\left(m_{\beta}\right)_{\beta \in B}$ such that $\left\{m_{\pi} \mid \pi \in \Pi\right\} \cup\left\{m_{\beta} \mid \beta \in B\right\}$ is a maximal farmily of mutually singular measures. (This can be done using Zorn's lemma.) An application of the Radon-Nikodym theorem yields:

$$
M(X)=\mathscr{C}(X)^{*}=\left(\sum_{\alpha \in \| l \cup B} \oplus L^{1}\left(m_{\alpha}\right)\right)_{l_{1}} .
$$


For each $\pi$ define $N_{\pi}$ as the set $\left\{\pi \in L^{1}\left(m_{\pi}\right) \mid \mu \perp A\right\}$. The abstract F. and M. Riesz theorem [6] and hypothesis 2 give that

$$
A^{\perp}=\left(\sum_{\pi \in I I} \oplus N_{\pi}\right)_{l_{1}}
$$

and hence

$$
A^{*}=\left(\sum_{\pi \in I I} \oplus L^{1}\left(m_{\pi}\right) / N_{\pi}\right)_{l_{1}} \oplus\left(\sum_{\beta \in B} \oplus L^{1}\left(m_{\beta}\right)\right)_{l_{1}} .
$$

In [2] and [1] it is proved that the spaces $L^{1}\left(m_{\pi}\right) / N_{\pi}$ have the Dunford-Pettis property. By the preceding lemma and Grothendieck's result that an $L^{1}$ space is a Dunford-Pettis space we have that $A^{*}$ has the D.P. property.

REMARK. (1) If $D=\{z|| z \mid<1\}$ and $A$ is the so-called discalgebra i.e. $A=\{f \mid f$ analytic on $D$, continuous on $\bar{D}\}$ then $A$ satisfies all requirements hence $A$ and $A^{*}$ have the D.P. property.

(2) If $K$ is a compact set which is finitely connected then by Wilken's theorem $R(K)$ satisfies hypothesis 2 and by [6, p. 145, paragraph 3], $R(K)$ also satisfies hypothesis 1 . Consequently $R(K)$ as well as $R(K)^{*}$ have the Dunford-Pettis property.

\section{REFERENCES}

1. Chaumat, Une généralisation d'un théorème de Dunford-Pettis, (preprint) Université Paris XI, Orsay, $\mathrm{n}^{\circ}$ 95, 1974.

2. Cnop-Delbaen, A Dunford-Pettis theorem for $L^{1} / H^{\infty}$. (to be published) preprint Department of Mathematics, Vrije Universiteit Brussel, Belgium 1975.

3. Dunford-Schwartz, Linear Operators, Part I, Interscience, New York, 1958.

4. Grothendieck, Sur les applications linéalires faiblement compactes sur les espaces du type $\mathscr{C}(K)$, Canad. J. of Math., (1953), 129-173.

5. — Espaces Vectoriels Topologique, São Paulo, 1964.

6. Gamelin, Uniform Algebras, Prentice-Hall, Englewood Cliffs, 1969.

Received August 4, 1975 and in revised form November 20, 1975.

VRIJe Universiteit BRussel 



\section{PACIFIC JOURNAL OF MATHEMATICS}

\section{EDITORS}

RICHARD ARENS (Managing Editor) University of California

Los Angeles, California 90024

R. A. Beaumont

University of Washington

Seattle, Washington 98105
J. DUGUNDJI

Department of Mathematics University of Southern Californı

Los Angeles, California 90007

D. Gilbarg and J. Milgram

Stanford University

Stanford, California 94305

\section{ASSOCIATE EDITORS}
E. F. BECKENBACH
B. H. NeumanN
F. WOLF
K. YosHIDA

\section{SUPPORTING INSTITUTIONS}

UNIVERSITY OF BRITISH COLUMBIA

CALIFORNIA INSTITUTE OF TECHNOLOGY

UNIVERSITY OF CALIFORNIA

MONTANA STATE UNIVERSITY

UNIVERSITY OF NEVADA

NEW MEXICO STATE UNIVERSITY

OREGON STATE UNIVERSITY

UNIVERSITY OF OREGON

OSAKA UNIVERSITY
UNIVERSITY OF SOUTHERN CALIFORNIA

STANFORD UNIVERSITY

UNIVERSITY OF TOKYO

UNIVERSITY OF UTAH

WASHINGTON STATE UNIVERSITY

UNIVERSITY OF WASHINGTON

* * * *

AMERICAN MATHEMATICAL SOCIETY

NAVAL WEAPONS CENTER 


\section{Pacific Journal of Mathematics \\ Vol. 65, No. $1 \quad$ September, 1976}

David Lee Armacost, Compactly cogenerated LCA groups ............. 1

Sun Man Chang, On continuous image averaging of probability measures ...... 13

J. Chidambaraswamy, Generalized Dedekind $\psi$-functions with respect to a

polynomial. II................................... 19

Freddy Delbaen, The Dunford-Pettis property for certain uniform algebras ..... 29

Robert Benjamin Feinberg, Faithful distributive modules over incidence

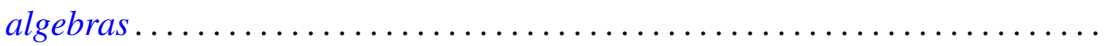

Paul Froeschl, Chained rings . . . . . . . . . . . . . . . . . . . .

John Brady Garnett and Anthony G. O'Farrell, Sobolev approximation by a sum

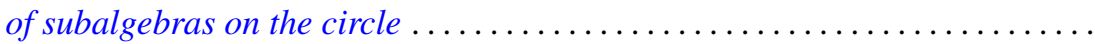

Hugh M. Hilden, José M. Montesinos and Thomas Lusk Thickstun, Closed

oriented 3-manifolds as 3-fold branched coverings of $S^{3}$ of special type.....

Atsushi Inoue, On a class of unbounded operator algebras ................

Peter Kleinschmidt, On facets with non-arbitrary shapes.

Narendrakumar Ramanlal Ladhawala, Absolute summability of Walsh-Fourier

series

Howard Wilson Lambert, Links which are unknottable by maps . . . . . . . . . . .

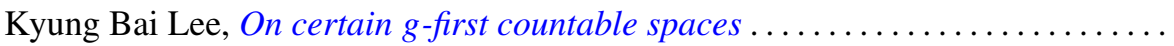

Richard Ira Loebl, A Hahn decomposition for linear maps .................

Moshe Marcus and Victor Julius Mizel, A characterization of functionals on $W_{1}^{p}$ possessing autonomous kernels. I . .

James Miller, Subordinating factor sequences and convex functions of several

variables.

Keith Pierce, Amalgamated sums of abelian l-groups ...

Jonathan Rosenberg, The $C^{*}$-algebras of some real and $p$-adic solvable

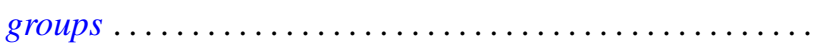

Hugo Rossi and Michele Vergne, Group representations on Hilbert spaces defined

in terms of $\partial_{b}$-cohomology on the Silov boundary of a Siegel domain . .

Mary Elizabeth Schaps, Nonsingular deformations of a determinantal

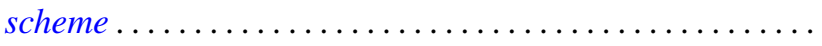

S. R. Singh, Some convergence properties of the Bubnov-Galerkin method...

Peggy Strait, Level crossing probabilities for a multi-parameter Brownian

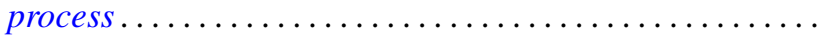

Robert M. Tardiff, Topologies for probabilistic metric spaces .

Benjamin Baxter Wells, Jr., Rearrangements of functions on the ring of integers of

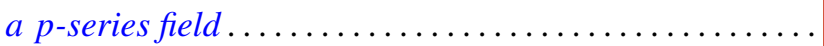

Robert Francis Wheeler, Well-behaved and totally bounded approximate identities for $C_{0}(X)$.

Delores Arletta Williams, Gauss sums and integral quadratic forms over local

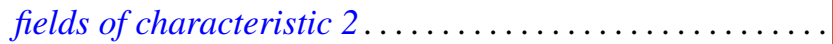

John Yuan, On the construction of one-parameter semigroups in topological 